PROCEEDINGS OF THE

AMERICAN MATHEMATICAL SOCIETY

Volume 126, Number 11, November 1998, Pages 3183-3189

S 0002-9939(98)04745-5

\title{
BOUNDS ON THE ORDER OF CROSS CHARACTERISTIC SUBGROUPS OF THE FINITE SIMPLE GROUPS OF LIE TYPE
}

\author{
TIHOMIR ASPAROUHOV
}

(Communicated by Ronald M. Solomon)

\begin{abstract}
Let $X(r)$ and $G(q)$ be finite groups of Lie type and $r$ and $q$ be coprime. If $G(q)$ is embedded in $X(r)$, then the Landazuri-Seitz-Zalesskii theorem implies that $G(q)$ is small relative to $X(r)$. We formalize this observation and illustrate how it can be used with some applications.
\end{abstract}

\section{INTRODUCTION}

If $X$ is a finite group of Lie type over a field of order $r$ and $G$ is a maximal subgroup of $X$, then it is essentially known that either $G$ is a member of one of several nice families of subgroups of $X$ or $G$ is an almost simple group. If $G$ is a finite simple group of Lie type over a field of order $q$ with $(q, r)=1$, then by the Landazuri-Seitz-Zalesskii theorem, the degree $N$ of the minimal nontrivial representation of $G$ over the nonnatural characteristic is very large compared to the degree of the minimal nontrivial representation of $G$ over the natural characteristic. This fact has been used implicitly in various places in the literature to show that $|X: G|$ is very large. In this note we formalize this observation and make it explicit in Propositions 1 and 2. Then we illustrate the strength of the observation with four applications. For the most part these applications are not new. They are intended to give the reader a sense of how Propositions 1 and 2 could be used.

Notice that the lower bound on $|X: G|$ is large when $N$ is large. On the other hand when $N$ is small, one can hope to enumerate all cross characteristic subgroups of $X$.

Liebeck and Saxl, in [L] and [LS], have obtained bounds on the orders of the almost simple maximal subgroups of a finite simple group of Lie type, which enabled them to list all maximal subgroups of large order. They proved that when $X$ is a classical group with natural projective module $V$ of dimension $n$ over $G F(r)$, then an almost simple maximal subgroup of $X$ is of order less then $r^{3 n}$, with some known exceptions. The bound $n^{12 \log _{2} n}$, that we obtain in Proposition 2 for cross characteristic subgroups is stronger, in fact this bound is independent of $r$. The situation for the exceptional groups is similar.

Received by the editors April 1, 1997.

1991 Mathematics Subject Classification. Primary 20E32.

(C)1998 American Mathematical Society 


\section{MAin RESUlt}

The following lemma is Lemma $2.1 \mathrm{in}$ [TZ].

Lemma 1. If $2 \leq r, 2 \leq a_{1}<a_{2}<\ldots<a_{k}$ are integers and $\epsilon_{1}, \epsilon_{2}, \ldots, \epsilon_{k} \in\{1,-1\}$, then

$$
\frac{1}{2} \leq \frac{\left(r^{a_{1}}+\epsilon_{1}\right)\left(r^{a_{2}}+\epsilon_{2}\right) \ldots\left(r^{a_{k}}+\epsilon_{k}\right)}{r^{a_{1}+a_{2}+\ldots+a_{k}}} \leq 2 .
$$

Let $G$ be a simple algebraic group over an algebraically closed field of characteristic $p$. Let $\sigma$ be a surjective homomorphism $G \rightarrow G$ such that $G_{\sigma}$ is finite. The finite groups $O^{p^{\prime}}\left(G_{\sigma}\right)$ obtained this way are the finite groups of Lie type in characteristic $p$.

Lemma 2. If $X(r)$ is a finite simple group of Lie type, then

$$
r^{d-2} \leq|X(r)| \leq r^{d+1},
$$

where $d$ is the dimension $\bar{d}$ of the corresponding algebraic simple group except for ${ }^{2} B_{2}(r),{ }^{2} G_{2}(r)$ and ${ }^{2} F_{4}(r)$, where $d=\bar{d} / 2$ (see the table, $r$ is a power of a prime p). The lemma holds for $\mathrm{PSp}_{4}(2)^{\prime}, G_{2}(2)^{\prime}$ and ${ }^{2} F_{4}(2)^{\prime}$ too.

\begin{tabular}{|l|l|l|}
\hline$X(r)$ & $d$ & $N$ \\
\hline$P S L_{n}(r)$ & $n^{2}-1$ & $n$ \\
\hline$P S p_{2 n}(r), n \geq 2$ & $2 n^{2}+n$ & $2 n$ \\
\hline$P S U_{n}(r), n \geq 3$ & $n^{2}-1$ & $n$ \\
\hline$P O_{2 n}^{+}(r), n \geq 4$ & $2 n^{2}-n$ & $2 n$ \\
\hline$P O_{2 n}^{-}(r), n \geq 4$ & $2 n^{2}-n$ & $2 n$ \\
\hline$P O_{2 n+1}(r), n \geq 3, r-$ odd & $2 n^{2}+n$ & $2 n+1$ \\
\hline$E_{6}(r),{ }^{2} E_{6}(r)$ & 78 & 27 \\
\hline$E_{7}(r)$ & 133 & 56 \\
\hline$E_{8}(r)$ & 248 & 248 \\
\hline$S z\left(2^{2 k+1}\right)$ & 5 & 4 \\
\hline${ }^{3} D_{4}(r)$ & 28 & 8 \\
\hline${ }^{2} F_{4}\left(2^{2 k+1}\right)$ & 26 & 26 \\
\hline${ }^{2} G_{2}\left(3^{2 k+1}\right)$ & 7 & 7 \\
\hline$F_{4}(r)$ & 52 & $26-\delta_{p, 3}$ \\
\hline$G_{2}(r)$ & 14 & $7-\delta_{p, 2}$ \\
\hline
\end{tabular}

Proof. If $X(r) \neq{ }^{3} D_{4}(q)$ we know that

$$
|X(r)|=\frac{r^{a}}{s}\left(r^{a_{1}}+\epsilon_{1}\right)\left(r^{a_{2}}+\epsilon_{2}\right) \ldots\left(r^{a_{k}}+\epsilon_{k}\right),
$$

for some integers $1 \leq a_{1}<a_{2}<\ldots<a_{k}$, such that $a+a_{1}+\ldots+a_{k}=d$. The number $s$ is the order of the center of the simply connected group of type $G(r)$. If $a_{1}>1$, then by Lemma 1 we get

$$
\frac{r^{d}}{2 s} \leq|X(r)| \leq \frac{2 r^{d}}{s} \leq r^{d+1}
$$

If $a_{1}=1$, then $G(r)$ is ${ }^{2} B_{2}(r),{ }^{2} G_{2}(r)$ or ${ }^{2} F_{4}(r)$ and $r=r_{1}^{l}$ for $l>1$. Now we can apply Lemma 1 for $r_{1}$ instead of $r$ to get the same result. If $X(r) \neq{ }^{2} E_{6}(2)$ and 
$X(r) \neq P S U_{n}(2)$ we can easily check that $r^{2} \geq 2 s$ and this proves the lemma. If $X(r)=P S U_{n}(2)$, then $s \leq 3$ and therefore

$$
\left|P S U_{n}(2)\right| \geq 2^{\frac{n(n-1)}{2}} \prod_{i=3}^{n}\left(2^{i}-(-1)^{i}\right) \geq 2^{n^{2}-3}=2^{d-2},
$$

because $\left(2^{i}+1\right)\left(2^{i+1}-1\right) \geq 2^{2 i+1}$. If $X(r)$ is ${ }^{2} E_{6}(2)$ or ${ }^{3} D_{4}(q)$ the Lemma is easily verified.

Proposition 1. Let $G(q)$ be a finite simple group of Lie type embedded in the group $P G L_{n}(K)$, where $K$ is a field of characteristic coprime to $q$. Then

$$
|G(q)| \leq n^{10+2 \log _{q} n} .
$$

Proof. This is a corollary of the Landazuri-Seitz-Zalesskii theorem [SZ] that gives a lower bound for $n$. Let $G(q)$ be $P S L_{m}(q)$ or $P S U_{m}(q)$. Then $n \geq q^{m-2}$ and therefore $m \leq \log _{q} n+2$. Also $d=m^{2}-1$ and by Lemma 2

$$
|G| \leq q^{d+1}=q^{m^{2}} \leq q^{\left(\log _{q} n\right)^{2}+2 \log _{q} n+4}=n^{\log _{q} n+2} q^{4} \leq n^{\log _{q} n+10} .
$$

The last step is true since $m \geq 3$ implies $n \geq q$, and if $m=2$, then $n \geq(q-1) / 2$ and $q \leq 2 n+1 \leq n^{2}$ except for $n=2$ and $q=5$, when the proposition is obvious. Let $G(q)$ be $P O_{m}(r)$ ( $m$ and $r$ odd), $P S p_{m}(r)$ ( $m$ even) or $P O_{m}^{ \pm}(r)$ ( $m$ even). Then $n \geq q^{m / 2-1} \geq q$ and $d \leq\left(m^{2}+m\right) / 2$. Now we get that $m \leq 2 \log _{q} n+2$ and

$$
|G| \leq q^{\left(m^{2}+m\right) / 2+1} \leq q^{\left(\left(2 \log _{q} n+2\right)^{2}+2 \log _{q} n+2\right) / 2+1}=n^{2 \log _{q} n+5} q^{4} \leq n^{2 \log _{q} n+9} .
$$

Finally if $G(q)$ is an exceptional finite simple group of Lie type, then $n \geq q^{(d+1) / 10}$. For example if $G(q)=E_{8}(q)$, then the LSZ theorem says that $n \geq q^{27}\left(q^{2}-1\right) \geq$ $q^{26} \geq q^{24.9}$. Similarly for all the exceptional groups. We get that $|G(q)| \leq q^{d+1} \leq$ $n^{10}$. Now we can easily check that the claim of the proposition holds for all the exceptions of the LSZ theorem and the proof is complete.

Proposition 2. Let $G(q) \leq X(r)$ be two finite simple groups of Lie type and $(r, q)=1$. Then

$$
|G(q)| \leq N^{12 \log _{q} N}
$$

and

$$
|X(r): G(q)| \geq r^{\left(d-2-12 \log _{r} N \cdot \log _{q} N\right)},
$$

where $d$ and $N$ are from the table. In fact, $N$ is the minimal degree of the representations of $X(r)$ over a field of the natural characteristic.

Proof. From the LSZ theorem if $G(q) \neq P S L_{2}(q)$, then $N \geq q$ and by Proposition 1

$$
|G(q)| \leq N^{10+2 \log _{q} N} \leq N^{12 \log _{q} N} .
$$

If $G=P S L_{2}(q)$, then $N^{2} \geq q$ and $|G(q)| \leq N^{6} \leq N^{12 \log _{q} N}$. The second part follows from the first part and Lemma 2 . 


\section{Applichtions}

A factorization of a finite group $G$ is an expression $G=A B$ for some proper subgroups $A$ and $B$ of $G$. The factorization is maximal if both $A$ and $B$ are maximal. The maximal factorizations of the finite classical groups are found in [LPS]. Here we consider factorizations of a finite classical group by a group of Lie type in a different characteristic. We obtain restrictions for such factorizations.

Proposition 3. Let $G(q)$ be a finite simple group of Lie type and $X_{n}(r)$ be one of the finite classical groups $P S L_{n}(r), P S U_{n}(r)(n \geq 3), P O_{n}(r)(n \geq 7$ and $r$ odd), $P S p_{n}(r)$ ( $n$ even $\left.\geq 4\right)$, or $P O_{n}^{ \pm}(r)$ ( $n$ even $\geq 8$ ) with $(q, r)=1$. If

$$
X_{n}(r)=H \cdot G(q)
$$

is a factorization of $X_{n}(r)$, then $G(q)$ is one of the following groups:

$P S L_{2}(q), q \leq 31 ; P S L_{3}(q), q \leq 4 ; P S L_{4}(2) ; P S p_{4}(q), q=3,5,7 ; P S p_{6}(q), q=2,3$;

$P S p_{8}(3) ; P S p_{4}(2)^{\prime} ; P S U_{3}(q), q=3,4 ; P S U_{4}(q), q=2,3 ; P S U_{5}(2) ; P O_{8}^{+}(2)$;

$\mathrm{PO}_{7}(3) ; G_{2}(q), q=3,4 ; S z(8)$. In addition, $r$ and $n$ must satisfy one of the following: $r=2$ and $n \leq 57 ; 2<r \leq 16$ and $r+n \leq 23 ; 16<r \leq 23$ and $n \leq 8$; $23<r \leq 29$ and $n \leq 4$; or $29<r \leq 59$ and $n=2$.

Proof. The smallest index of a subgroup of $X_{n}(r)$ is found in [Co] and is at least $r^{n-2}$. Therefore

$$
n^{10+2 \log _{q} n} \geq|G(q)| \geq\left|X_{n}(r): H\right| \geq r^{n-2} .
$$

The above inequality is not true if $n \geq 137$ for any $r$ and $q,(r, q)=1$. Therefore $n \leq 136$. Let $l(G(q))$ be the minimal degree of the representations of $G(q)$ over a field of cross characteristic. Then the group $G(q)$ is restricted to the groups that satisfy the following condition: $l(G(q)) \leq 136$ and $|G(q)| \geq r^{n-2} \geq r^{l(G(q))-2}$ or if $q$ is even then $|G(q)| \geq 3^{l(G(q))-2}$ and if $q$ is odd then $|G(q)| \geq 2^{l(G(q))-2}$. The above list of groups consists of the finite simple groups of Lie type satisfying these two conditions. Now for a fixed $r$ the possibilities for $n$ are restricted by the following

$$
l(G(q)) \leq n \leq \log _{r}|G(q)|+2
$$

for at least one of the groups $G(q),(q, r)=1$ in the above list. Let $d(G(q))$ be the minimal degree of a nontrivial projective representation of $G(q)$ over the field of complex numbers. For the finite classical groups $d(G(q))$ can be found in [TZ]. If $(r,|G(q)|)=1$, then additional restriction for $n$ is $d(G(q)) \leq n$. Now if $r=2$, the inequalities in (1) amount to $n \leq 57$. Similarly if $r=3$ we get $n \leq 20$ and so on for $r=4, \ldots, 16$. If $r>16$ we get $n \leq 8$. If $r>23$ we get $n \leq 6$. If $n \leq 6$ and $r>11$, then by [Co] the smallest index of a subgroup of $X_{n}(r)$ is at least $\left(r^{n}-1\right) /(r-1)$ and therefore $n$ is restricted to

$$
\left(r^{n}-1\right) /(r-1) \leq|G(q)| .
$$

Now by (1) and (2), if $r>23$ then $n \leq 4$. Finally if $r>29$ then $n$ must be 2 . There are two possibilities: $G=P S L_{2}(4)$ and $r+1 \leq|G|=60$, or $G=P S L_{3}(2)$, $r+1 \leq|G|=168$ and $(r,|G|) \neq 1$ (since $d(G)>2$ ). These imply the final statement.

Lemma 3. Let $G=X_{n}(r)$ be a finite classical group as in Proposition 3. If $G$ acts transitively on a set $\Omega$ and the stabilizer of a point is isomorphic to a finite simple group of Lie type $H=G(q)$ for $(q, r)=1$, then 
(1) for any element $1 \neq g \in G$, the fixed point ratio

$$
\frac{f(g)}{|\Omega|} \leq \frac{n^{10+2 \log _{q} n}}{r^{n-2}}
$$

where $f(g)$ is the number of elements of $\Omega$ fixed by $g$;

(2) the permutation rank of $G$ on $\Omega$ satisfies

$$
\operatorname{rank}(G) \geq r^{n^{2} / 4-24 \log _{r} n \log _{q} n} .
$$

Proof. By Proposition 1, the fixed point ratio

$$
\frac{f(g)}{|\Omega|}=\frac{\left|g^{G} \cap H\right|}{\left|g^{G}\right|} \leq \frac{|H|}{\left|G: C_{G}(g)\right|} \leq \frac{n^{10+2 \log _{q} n}}{r^{n-2}} .
$$

Also $|\Omega| \leq \operatorname{rank}(G)|H|$ and therefore

$$
\operatorname{rank}(G) \geq \frac{|G|}{|H|^{2}} \geq r^{d-2-24 \log _{q} n \log _{r} n} \geq r^{n^{2} / 4-24 \log _{r} n \log _{q} n} .
$$

Let $G$ be a transitive permutation group on the set $\Omega,|\Omega|=m$. For a permutation $g \in G$ let $\operatorname{orb}(g)$ be the number of orbits of $g$ on $\Omega$ and the index of $g$ be $\operatorname{Ind}(g)=m-\operatorname{orb}(g)$. A genus $l$ system is a triple $(G, \Omega, S)$, where $S=\left(g_{i} \neq 1: i=\right.$ $1, \ldots, r)$ is a generating set for $G$ such that $g_{1} g_{2} \ldots g_{r}=1$ and

$$
2(m+l-1)=\sum_{i=1}^{r} \operatorname{Ind}\left(g_{i}\right) .
$$

This condition is equivalent to the existence of a branched covering of the Riemann sphere by a Riemann surface of genus $l$ with monodromy group $G$. The study of genus 0 groups has been reduced in [A1], [GT] and [Sh] to the study of primitive almost simple groups $G$ such that $f\left(g_{i}\right) / m>1 / 85$ for some $i$.

Proposition 4. If $n>145$ the permutation group described in Lemma 3 is not a genus 0 group.

Proof. If $n>145$, then Lemma 3 (1) implies that for any $1 \neq g \in G$

$$
\frac{f(g)}{m}<\frac{1}{85} .
$$

Since $\operatorname{orb}(g)=\sum_{i=1}^{d} f\left(g^{i}\right) / d$, where $d$ is the order of $g$, we get that

$$
\operatorname{Ind}(g) \geq m-(d-1) m /(85 d)-m / d=\frac{84}{85} m(1-1 / d) .
$$

Now if $G$ is a genus 0 group with some $S=\left(g_{i}: i=1, \ldots, r ; d_{i}=\left|g_{i}\right|\right)$, then

$$
2 m-2=\sum_{i=1}^{r} \operatorname{Ind}\left(g_{i}\right) \geq \frac{84}{85} m\left(r-\sum_{i=1}^{r} 1 / d_{i}\right) .
$$

This inequality forces $r$ to be less than 5 , and if $r=4$ then $d_{1}=\ldots=d_{4}=2$ and if $r=3$ then $\sum 1 / d_{i} \geq 1$. Now (9.6) in [GT] states that $G$ is solvable or is isomorphic to $A_{5}$, which is impossible. 


\section{Bounds On THE SYlow SubGroups}

The following application of our main proposition was suggested by R. Guralnick. We want to formalize the observation that if a simple subgroup of a finite simple group of Lie type of characteristic $p$ has a large $p$-subgroup, then it has to be a group of Lie type of the same characteristic. For a prime number $p$ define $e_{p}(n)$ to be the maximal $i$ such that $p^{i}$ divides $n$ and let $\Phi_{k}(x)$ be the $k$-th cyclotomic polynomial. We will need the following well known number theory result; see $[\mathrm{M}]$ page 27 .

Lemma 4. Let $p$ be a prime number and $q$ be a power of a different prime number. Then $p \mid \Phi_{k}(q)$ if and only if $k=a \cdot p^{j}$, where $a$ is the minimal number $i$ such that $p \mid\left(q^{i}-1\right)$. In this case $e_{p}\left(\Phi_{a}(q)\right)=e_{p}\left(q^{a}-1\right)$ and for $j>0, e_{p}\left(\Phi_{a p^{j}}(q)\right)=1$, except for $e_{2}\left(\Phi_{2 a}(q)\right)=e_{2}(q+1)$ if $4 \mid(q+1)$.

Lemma 5. Let $j$ be $e_{p}\left(\prod_{i=1}^{n}\left(q^{i}-1\right)\right)$. Then $p^{j}<q^{p n /(p-1)}$.

Proof. Let $a$ be as in Lemma 4 . Then $p \mid q^{a}-1$ and therefore $p \leq q^{a}$. Since

$$
\prod_{i=1}^{n}\left(q^{i}-1\right)=\prod_{i=1}^{n} \Phi_{i}(q)^{\left[\frac{n}{i}\right]},
$$

we can use Lemma 4 to estimate the $p$-part of the above product. If $p>2$

$$
p^{j}<\left(q^{a}\right)^{[n / a]} \cdot\left(q^{a}\right)^{[n /(a p)]} \cdot\left(q^{a}\right)^{\left[n /\left(a p^{2}\right)\right]} \ldots \leq q^{n\left(1+p^{-1}+p^{-2}+\ldots\right)}<q^{p n /(p-1)} .
$$

When $p=2$ a similar calculation yields the result.

Lemma 6. Let $P$ be a cross characteristic Sylow subgroup of a finite simple classical group $X$. If $X=P S L_{n}(q)$, then $|P|<q^{2 n}$; if $X=O_{2 n+1}(q), P O_{2 n}^{ \pm}(q)$, $P S p_{2 n}(q)$, then $|P|<q^{4 n}$; and if $X=P S U_{n}(q)$, then $|P|<(q+1)^{2 n-1}$.

Proof. If $X \neq P S U_{n}(q)$, then this Lemma follows directly from Lemma 4 . If $X=P S U_{n}(q)$, then we need to apply Lemma 4 for $-q$ instead of $q$ and repeat the proof of the Lemma using $p \leq q^{a} \pm 1 \leq(q+1)^{a}$.

Lemma 7. Let $X(r), G(q)$ and $N$ be as in Proposition 2. Let $r=p^{l}, p$ a prime number. If $P$ is a $p$-Sylow subgroup of $G(q)$, then $|P|<N^{10}$.

Proof. If $G$ is an exceptional group, then from the proof of Proposition 1 we get $|P|<|G|<N^{10}$. If $G=P S U_{m}(q)$, then by Lemma $6|P|<(q+1)^{2 m-1}$ and as in the proof of Proposition 1, $m \leq \log _{q} N+2$ and we get

$$
|P|<(q+1)^{\left(2 \log _{q} N+3\right)}<\left(q^{2}\right)^{5 \log _{q} N}=N^{10} .
$$

Similar arguments apply for the rest of the classical groups.

Proposition 5. Let $X(r)$ and $N$ be as in Proposition 2, $r=p^{l}$ and $p$ be a prime number. Let $S$ be a simple subgroup of $X$ and let $P$ be its $p$-Sylow subgroup. If $|P| \geq N^{10}$, then $S$ is an alternating group or a group of Lie type of the same characteristic. If also $|P| \geq p^{(N+2) /(p-1)}$, then $S$ is a group of Lie type of the same characteristic.

Proof. It's easy to observe that $S$ is not a sporadic group. If $S$ is the alternating group $A_{m}$, then the minimal projective module is of dimension at least $m-2$. Finally

$$
|P|<p^{m /(p-1)} \leq p^{(N+2) /(p-1)} .
$$




\section{REFERENCES}

[A1] M. Aschbacher, "On Conjectures of Guralnick and Thompson" J. Algebra 135 (1990) 277343. MR 91m:20007

[Co] B. Cooperstein , "Minimal Degree for a Permutation Representation of a Classical Group" Israel J. Math. 30 (1978), 213-235. MR 58:22255

[GT] R. Guralnick and J. Thompson, "Finite Groups of Genus Zero" J. Algebra 131 (1990) 303-341. MR 91e:20006

[L] M. Liebeck, "On the orders of maximal subgroups of finite classical groups", Proc LMS (3) (1985), 426-446. MR 87a:20046

[LPS] M. Liebeck, C. Praeger and J. Saxl, "The Factorization of the Finite Simple Groups and Their Automorphism Groups" Mem. of AMS v86 (432) (1990), 1-151. MR 90k:20048

[LS] M. Liebeck and J. Saxl, "On the orders of maximal subgroups of exceptional groups of Lie type", Proc LMS(2) 31 (1985), 237-249.

[M] K. Motose, "On Values of Cyclotomic Polynomials" II, Math J. of Okayama Univ. vol. 37 (Dec. 1995), 27-36. MR 97h:11151

[Sh] T. Shih, "A Note on Groups of Genus Zero", Comm. Algebra 10 (1991) 2813-2826. MR 93b:20009

[SZ] G. Seitz and A. Zalesskii, "On the Minimal Degree of Projective Representations of the Finite Chevalley Groups, II" J. Algebra 158 (1993), 233-243. MR 94h:20017

[TZ] P. Tiep and A. Zalesskii, "Minimal Characters of the Finite Classical Groups" Comm. Algebra 24 (1996), 2093-2167. MR 97f:20018

California Institute of Technology, Pasadena, California 91125

E-mail address: tihomir@cco.caltech.edu 